\title{
Dissipative electromagnetism from a nonequilibrium thermodynamics perspective
}

\author{
Asja Jelić, Markus Hütter, and Hans Christian Öttinger \\ Department of Materials, Institute of Polymers, \\ ETH Zurich, 8093 Zurich, Switzerland.
}

(Dated: April 19, 2022)

\begin{abstract}
Dissipative effects in electromagnetism on macroscopic scales are examined by coarse graining the microscopic Maxwell equations with respect to time. We illustrate a procedure to derive the dissipative effects on the macroscopic scale by using a Green-Kubo type expression in terms of the microscopic fluctuations and the correlations between them. The resulting macroscopic Maxwell equations are formulated within the General Equation for the Non-Equilibrium ReversibleIrreversible Coupling (GENERIC) framework, accounting also for inhomogeneous temperature.
\end{abstract}

PACS numbers: 05.70.Ln, 03.50.De, 05.40.-a 


\section{INTRODUCTION}

The dynamics of matter can be described on different levels of detail. For example, microscopic descriptions resolve short length scales and fast processes. In principle, one can follow the microscopic dynamics over very long times to arrive at the behavior on the macroscopically relevant time scales. However, doing so is often not desirable, and one can learn significantly more by using a coarse-grained description. For example, the flow of water in a complex geometry is usually studied not in terms of the dynamics of water molecules, but rather in terms of the continuum equations of hydrodynamics. This is so because key features of interest emerge only on the length and time scales much larger than the molecular ones. The intrinsic time scale for the relaxation of inhomogeneities in the macroscopic velocity field is closely related to the shear viscosity $\eta$. This material specific property can either be determined experimentally on purely macroscopic grounds. Another, conceptually very interesting, route goes via the so-called fluctuation-dissipation theorem $\underline{1}$ 4], which establishes a relation between micro- and macroscopic properties. In particular, the Green-Kubo formula relates the shear viscosity to the correlations between fluctuations of the microscopic shear stress, $\sigma_{x y}$, by way of

$$
\eta=\frac{V}{k_{\mathrm{B}} T} \int_{0}^{\infty}\left\langle\sigma_{x y}(t) \sigma_{x y}(0)\right\rangle_{\mathrm{eq}} d t
$$

with $V$ the sample volume, $T$ the absolute temperature, and $\langle\ldots\rangle_{\text {eq }}$ the equilibrium average. The integration range $[0, \infty]$ stands symbolically for an integration range much longer than any microscopic relaxation time. However, it is implicitly assumed that this range is shorter than macroscopic time scales, which implies that micro- and macroscopic timescales are clearly separated. Relations of this type are frequently used in molecular dynamics simulations to determine macroscopic transport properties [3], where transport coefficients are expressed in terms of two-time correlations of current densities.

The motivation for the work presented here related to the Maxwell equations rests on the following idea. The Green-Kubo relation (1), and generalizations thereof, not only tells us how to relate microscopic fluctuations to macroscopic transport coefficients. More fundamentally, it indicates that microscopic fluctuations give rise to dissipative processes in more coarse-grained descriptions. In view of electromagnetism, this serves as a strong 
motivation to believe that, e.g., fluctuations in the polarization and magnetization by way of particle vibrations and fast spin dynamics must be observable on macroscopic scales as dissipative processes. Although the Maxwell equations belong to the most studied differential equations in physics, they do not account for the dissipative phenomena beyond Ohmic resistance and the frequency dependent imaginary parts of the permittivity and permeability in the linear response regime. In view of the above discussion, we anticipate that the electric (Ohmic) resistance is related to the fluctuations of the electric current of the unbound charges. However, in addition also the bound particles fluctuate, giving rise to fluctuations in the polarization and the magnetization. How those fluctuations give rise to dissipative effects on the macroscopic scale is precisely what we want to elaborate on in this manuscript.

Thermodynamics comes into play in the Green-Kubo relation (1) by way of the absolute temperature $T$. In addition, the occurrence of a "thermal" variable is also of fundamental importance in a purely macroscopic model with dissipative effects, since the latter lead to an entropy increase, and in turn to a change in the thermal state. In contrast, the Maxwell equations are usually taken as a set of isolated equations, decoupled from the other macroscopic variables, e.g., temperature and density. This means that the evolution of the electromagnetic field is generally obtained in the approximation that these macroscopic variables have set values or, at best, are given as functions of time. In order to obtain a rigorous and reliable theory, one needs to include these macroscopic quantities in the list of variables and to consider their dynamics in conjunction with the time evolution of the electromagnetic field. Only then one can capture the interplay between the thermodynamic behavior and the electromagnetic field, and dissipative effects in electromagnetism can be addressed in a consistent manner.

To perform a thorough theoretical analysis of the interplay between the thermodynamic properties and the electromagnetic field is a demanding task, especially if one wants to account for dissipation effects beyond a linear response theory. Liu and coworkers have incorporated dissipative effects into the Maxwell equations [5-11]. The resulting theory describes the dynamics of macroscopic systems that are exposed to electromagnetic fields and contain electric charges and currents. In particular, the driving forces for the dissipative effects on the macroscopic scale have been established. In the dynamic equations, the new dissipative terms occur as an addition to the commonly used macroscopic Maxwell equations when the system is out of equilibrium. The validity of this theory was at first confined to 
the low-frequency regime, but was later generalized to higher frequencies [11].

In this paper the goal is to use the reversible Maxwell equations to derive macroscopic Maxwell equations for dissipative electromagnetism in a medium, valid also outside of the linear response regime. As the guideline to complete this task, the General Equation for the Non-Equilibrium Reversible-Irreversible Coupling (GENERIC) formalism of non-equilibrium

thermodynamics [4, 12, 13] is used for two reasons. First, thermodynamic consistency of the macroscopic model is ensured. And second, the formalism is equipped with a scheme for temporal coarse-graining, the most important part of which is concerned with the emergence of dissipative effects upon coarse-graining, i.e., a generalization of the Green-Kubo relation (1). This scheme will be illustrated below specifically on the Maxwell equations in order to obtain the macroscopic equations that possess the GENERIC structure. These equations are then compared to the dissipative Maxwell equations suggested by Liu [5].

The paper is organized as follows. In Sec. II , the microscopic and common macroscopic Maxwell equations are briefly presented. The usual coarse-graining procedure and its shortcomings are recapitulated. In Sec. III, a GENERIC formulation of the Maxwell equations together with the equation for the energy density is presented. We illustrate the procedure to obtain, by temporal coarse-graining using Green-Kubo-type expressions, the dissipative effects in the time evolution equations for the macroscopic variables.

\section{MICRO- AND MACROSCOPIC MAXWELL EQUATIONS}

The classical derivation of the macroscopic Maxwell equations for the electromagnetic fields in a medium from the microscopic ones,

$$
\begin{array}{rlrl}
\dot{\mathbf{b}} & =-\nabla \times \mathbf{e}, & \nabla \cdot \mathbf{b} & =0, \\
\dot{\mathbf{e}} & =\frac{1}{\epsilon_{0} \mu_{0}} \nabla \times \mathbf{b}-\frac{1}{\epsilon_{0}} \mathbf{j}, & \nabla \cdot \mathbf{e}=\frac{1}{\epsilon_{0}} \rho,
\end{array}
$$

is often performed through spatial averaging [14]. Throughout the manuscript we denote the

partial time derivative by $\dot{\mathbf{A}} \equiv \frac{\partial}{\partial t} \mathbf{A}$. The quantities $\epsilon_{0}$ and $\mu_{0}$ stand for the permittivity and the permeability, respectively, in vacuum. Starting from the microscopic Maxwell equations containing only fields $\mathbf{e}$ and $\mathbf{b}$, one separates the free from the bound parts of the charge $\rho$ and of the current density $\mathbf{j}$. Upon spatial averaging, the expressions for the bound parts of $\rho$ and $\mathbf{j}$ are simplified by introducing the polarization $\mathbf{P}$ and the magnetization $\mathbf{M}$. With $\mathbf{E}$ and 
B the spatially averaged electric field and magnetic induction, respectively, the macroscopic Maxwell equations can then be formulated conveniently as

$$
\begin{array}{ll}
\dot{\mathbf{B}}=-\nabla \times \mathbf{E}, & \nabla \cdot \mathbf{B}=0, \\
\dot{\mathbf{D}}=\nabla \times \mathbf{H}-\mathbf{J}_{e}, & \nabla \cdot \mathbf{D}=\rho_{e},
\end{array}
$$

where one has introduced the electric displacement $\mathbf{D}=\epsilon_{0} \mathbf{E}+\mathbf{P}$, assuming that quadrupol moments are negligible, and the magnetic field $\mathbf{H}=\mathbf{B} / \mu_{0}-\mathbf{M}$. Doing so, the source terms of the macroscopic Maxwell equations are given by the averages of the free charge density and free current density, $\rho_{e}$ and $\mathbf{J}_{e}$, respectively. In addition, the evolution equation for the energy density of the electromagnetic field can be written in the form

$$
\dot{\varepsilon}_{\mathrm{em}}=-\nabla \cdot(\mathbf{E} \times \mathbf{H})-\mathbf{J}_{e} \cdot \mathbf{E},
$$

where $\mathbf{E} \times \mathbf{H}$ is the Poynting vector.

The structure of the equations (3) imposes the interpretation that the field variables are $\mathbf{D}$ and $\mathbf{B}$, the temporal equations are their time evolution equations, while the other two represent the constraints that must be satisfied at all times. The fields $\mathbf{E}$ and $\mathbf{H}$ then need to be expressed as some functions of $\mathbf{D}$ and $\mathbf{B}$ in order to render equations (3) closed. Such closing relations are known as constitutive equations, which contain the information about the medium, and are usually considered as given in macroscopic electrodynamics. The usual choice is to use linear relations between $(\mathbf{E}, \mathbf{B})$ and $(\mathbf{D}, \mathbf{H})$, valid if the electromagnetic field is sufficiently weak, in order to obtain the properties of the permittivity $\epsilon$ and permeability $\boldsymbol{\mu}$ that describe the response of a medium on the electromagnetic field. Then the real parts of the functions $\boldsymbol{\epsilon}(\omega)$ and $\boldsymbol{\mu}(\omega)$ express the oscillatory motion of the bound charges and the imaginary parts describe dissipation. However, in many cases, like the passage of the laser beam through a substance, or for a medium in a strong magnetic field, the linear response theory is not valid anymore and one needs to work in the regime of nonlinear electrodynamics, with the consequence of loosing all the simple relations.

A common constitutive relation concerns the current density. It is known that Ohmic conductors satisfy the condition $\mathbf{E}^{\mathrm{eq}}=\mathbf{0}$ at equilibrium, and that in non-equilibrium situations the force to relax $\mathbf{D}$ to equilibrium is proportional to $\mathbf{E}$. Such behavior is often expressed by $\mathbf{J}_{e}=\sigma \mathbf{E}$ with conductivity $\sigma$. This interrelation suggests to draw the following analogy. In stationary, equilibrium situations, with vanishing electric current $\mathbf{J}_{e}$, one obtains 
from (3) the conditions

$$
\nabla \times \mathbf{H}^{\mathrm{eq}}=\mathbf{0}, \quad \nabla \times \mathbf{E}^{\mathrm{eq}}=\mathbf{0}
$$

We therefore assume that in non-equilibrium situations on the macroscopic scale there are forces to drive $\mathbf{B}$ and $\mathbf{D}$ towards equilibrium with those forces being closely related to $\nabla \times \mathbf{H}$ and $\nabla \times \mathbf{E}$ with associated transport coefficients. Conditions (5) have been obtained also by Liu by minimizing the total energy of the system under the constraints of the non-temporal Maxwell equations $\nabla \cdot \mathbf{D}=\rho_{e}$ and $\nabla \cdot \mathbf{B}=0[\underline{5}]$.

The commonly used macroscopic Maxwell equations (3) can be obtained not only by spatial averaging (see e.g. [14]), which is also called "truncation" in [15], but also by ensemble averaging [15, 16]. In both cases, only single time properties are considered, while temporal correlations and two-time ensemble averages are neglected. In the remainder of this manuscript, we will thus refer to these two techniques as "spatial" averaging, in contrast to temporal coarse-graining which will be discussed in detail below. Neglecting coarse-graining with respect to time to arrive at the spatially averaged Maxwell equations (3) may be a valuable ansatz under many circumstances [14]. However, it is also known that coarse-graining with respect to space and time can lead to a better understanding, and some aspects of a model emerge clearly only on longer time scales [4, 17]. Such an example is hydrodynamics, where the shear viscosity given by (11) and other transport coefficients in the Navier-Stokes equations arise at the continuum level of description, but are absent in a molecular model. Therefore, we will examine specifically the effect of coarse-graining in time by using GreenKubo-type expressions to learn more about dissipative effects in electromagnetic systems with nonlinear constitutive relations. In order to obtain a closed set of the macroscopic time evolution equations for electromagnetism in a medium, a scheme for temporal coarsegraining of the "spatially averaged" Maxwell equations is presented following the GENERIC formalism [4, 12, 13]. The full coarse-graining procedure together with the expressions obtained for the additional dissipative terms is presented in the following sections.

\section{GENERIC FORMULATION}

\section{A. Formalism}

The main points of the GENERIC framework of nonequilibrium thermodynamics 
[4, 12, 13] can be summarized briefly in the following way. In analogy to equilibrium thermodynamics, major importance comes to, first, choosing a complete set of variables $\mathbf{x}$, which describes the situation of interest to the desired detail. The reversible contributions to the time evolution for this set of variables, $\left.\dot{\mathbf{x}}\right|_{\text {rev }}$, is formulated in close reference to classical Hamiltonian mechanics. In particular, it is related to the energy gradient by way of a Poisson operator $\mathbf{L}$, i.e., $\left.\dot{\mathbf{x}}\right|_{\mathrm{rev}}=\mathbf{L} \cdot(\delta E / \delta \mathbf{x})$. The Poisson bracket associated to $\mathbf{L}$, given by $\{A, B\}=(\delta A / \delta \mathbf{x}, \mathbf{L} \cdot \delta B / \delta \mathbf{x})$ with appropriate scalar product (...), must be antisymmetric and satisfy the Jacobi identity, which are both abstract features that capture the nature of reversibility. In order to formulate the irreversible part of the dynamics one is motivated by the reversible part. For the reversible dynamics, the energy plays a distinct role. First, it is a conserved quantity for closed systems and, second, it drives the reversible dynamics. In parallel, for irreversible dynamics entropy is a fundamentally important quantity, which must not decrease for closed systems. In analogy to the reversible dynamics, it is assumed in the GENERIC framework that the irreversible contributions to the time evolution of $\mathbf{x}$, $\left.\dot{\mathbf{x}}\right|_{\text {irrev }}$, is driven by the entropy gradient, i.e., that it is of the form $\left.\dot{\mathbf{x}}\right|_{\text {irrev }}=\mathbf{M} \cdot(\delta S / \delta \mathbf{x})$, with $\mathbf{M}$ a generalized friction matrix. The latter is required to be (Onsager-Casimir) symmetric, and contains transport coefficients and relaxation times associated to the corresponding dissipative effects. The condition that the friction matrix is positive semi-definite ensures that $\dot{S} \geq 0$ is fulfilled.

In summary, the time evolution of $\mathbf{x}$ can be expressed in terms of four building blocks $E$, $S, \mathbf{L}$ and $\mathbf{M}$ as

$$
\dot{\mathbf{x}}=\mathbf{L}(\mathbf{x}) \cdot \frac{\delta E(\mathbf{x})}{\delta \mathbf{x}}+\mathbf{M}(\mathbf{x}) \cdot \frac{\delta S(\mathbf{x})}{\delta \mathbf{x}} .
$$

The two different contributions to the time evolution, reversible and irreversible, are not independent. Rather, they are interrelated by the two degeneracy requirements

$$
\mathbf{L}(\mathbf{x}) \cdot \frac{\delta S(\mathbf{x})}{\delta \mathbf{x}}=\mathbf{0}, \quad \mathbf{M}(\mathbf{x}) \cdot \frac{\delta E(\mathbf{x})}{\delta \mathbf{x}}=\mathbf{0} .
$$

The first condition expresses the reversible nature of the $\mathbf{L}$ contribution to the dynamics, demonstrating the fact that the reversible dynamics captured in $\mathbf{L}$ does not affect the entropy functional. The second one expresses the conservation of the total energy of an isolated system by the irreversible contribution to the system dynamics captured in $\mathbf{M}$.

A particular feature of the GENERIC is that it is applicable on different levels of description, including reversible Hamiltonian mechanics and dissipative macroscopic field theories. 
The corresponding four building blocks obviously differ between the different levels. However, there are abstract procedures to relate them. Most importantly, the friction matrix on a coarse-grained level can be expressed in terms of the more microscopic dynamics [4]. This procedure helps to understand how dissipative effects can arise in coarse-grained descriptions. It is particularly this issue that we want to address in relation to the Maxwell equations of electrodynamics.

\section{B. Choice of variables, energy and entropy functionals}

First, we identify a natural set of variables for a dynamic description of dissipative electromagnetism. In order to describe a body in an electromagnetic field, we make the local equilibrium assumption, so that temperature, internal energy and entropy densities are defined at each point of the nonequilibrium system. Although one is free to choose any of these three variables for describing the "thermal" state of the body, it will become clear below that it is particularly useful to choose the energy density $\varepsilon$, since it is the density of a variable which is conserved for a closed system. The energy density $\varepsilon$ stands for the total energy density inside the system volume, consisting of both the internal energy of matter and the energy of the electromagnetic field. As far as the variables describing the electromagnetic state are concerned, we keep in mind that our primary interest is in the effect of coarse-graining the Maxwell equations with respect to time. Hence, we use the already spatially averaged fields D and B as used in the temporal Maxwell equations in (3). Since our focus here is on the temporal coarse-graining and on constructing the dissipative effects in a macroscopic formulation of electromagnetism, we consider only bodies at rest to avoid additional complexity, i.e., we do not include the velocity field in the set of variables. This restriction, in turn, also means that the mass density is a constant, as one can easily infer from the mass balance equation. In other words, we restrict our attention to materials for which the volume changes are negligible compared to the other effects of interest, namely materials with vanishingly small isothermal compressibility and thermal expansion coefficient. The full set of variables is thus given by $\mathbf{x}=(\varepsilon, \mathbf{D}, \mathbf{B})$. The total energy and entropy 
of the system, expressed in terms of these variables, are given by the functionals

$$
\begin{aligned}
& E=\int \varepsilon d^{3} r, \\
& S=\int s(\varepsilon, \mathbf{D}, \mathbf{B}) d^{3} r .
\end{aligned}
$$

The functional derivatives of $E$ and $S$ will be needed further below for the calculation of time evolution of variables $\mathbf{x}$ by using the general evolution equation (66). In order to connect the expressions for these functional derivatives to known quantities, one refers to the Gibbs thermodynamic relation. The latter gives the change in the energy density $\varepsilon$ of a thermally isolated medium at rest in the form of the total differential

$$
d \varepsilon=T d s+\mathbf{E} \cdot d \mathbf{D}+\mathbf{H} \cdot d \mathbf{B}
$$

under the assumption of local equilibrium, where the fields $\mathbf{E}$ and $\mathbf{H}$, as well as the temperature $T$, are given as the partial derivatives of the energy density $\varepsilon$ with respect to the appropriate variables. The first term on the right side in (9) is identical to the ordinary thermodynamic relation for the internal energy density in the absence of the electromagnetic field and for a constant mass density. The last two terms represent the change of the energy density due to changes in the electromagnetic fields. The form of these two terms is well known from the standard textbooks on electrodynamics [14, 18], where the total electromagnetic energy increment is given as a volume integral over the increments $\delta \mathbf{D}$ and $\delta \mathbf{B}$ multiplied by $\mathbf{E}$ and $\mathbf{H}$, respectively. Relation (9) is also in agreement with the total energy density used in [19] for a system at rest. We point out that the precise form of the energy density $\varepsilon$ of the system is not required below for the derivation of the macroscopic Maxwell equations in the GENERIC form.

The functional derivatives $\delta E / \delta \mathbf{x}$ and $\delta S / \delta \mathbf{x}$ are now obtained by reordering Eq.(9) in terms of the entropy total differential, and calculating the appropriate partial derivatives therefrom. One finds the following expressions needed for the GENERIC time evolution 
equation ([6):

$$
\begin{aligned}
& \frac{\delta E}{\delta \mathbf{x}}=\left(\begin{array}{l}
1 \\
\mathbf{0} \\
\mathbf{0}
\end{array}\right), \\
& \frac{\delta S}{\delta \mathbf{x}}=\frac{1}{T}\left(\begin{array}{c}
1 \\
-\mathbf{E} \\
-\mathbf{H}
\end{array}\right) .
\end{aligned}
$$

\section{Reversible dynamics}

The reversible contribution to the time evolution of the variables $\mathbf{x}=(\varepsilon, \mathbf{D}, \mathbf{B})$ is related to the energy gradient $\delta E / \delta \mathbf{x}$ by way of the Poisson operator $\mathbf{L}$. At this stage, we point out that the effects arising through coarse-graining in time by using the Green-Kubo formula are completely comprised in the friction matrix $\mathbf{M}$, i.e., in the irreversible part of the time evolution. Correspondingly, temporal coarse-graining does not affect the reversible part of the time evolution of the variables $\mathbf{x}$. Therefore, the Poisson operator

$$
\mathbf{L}=\left(\begin{array}{ccc}
L_{\varepsilon \varepsilon} & L_{\varepsilon \mathbf{D}} & L_{\varepsilon \mathbf{B}} \\
L_{\mathbf{D} \varepsilon} & L_{\mathbf{D D}} & L_{\mathbf{D B}} \\
L_{\mathbf{B} \varepsilon} & L_{\mathbf{B D}} & L_{\mathbf{B B}}
\end{array}\right)
$$

can be build up from the already established Maxwell equations (3) and the adequate evolution equation for the total energy density, given only by the first term in Eq. (4) for a medium at rest.

We approach the construction of $\mathbf{L}$ in the following manner, having in mind that the reversible contributions are of the form $\mathbf{L} \cdot(\delta E / \delta \mathbf{x})$. Since only the first element of the vector $\delta E / \delta \mathbf{x}$ is non-zero, Eq.(10a), terms $L_{\mathbf{D} \varepsilon}$ and $L_{\mathbf{B} \varepsilon}$ must reproduce the time evolution equations (3). The antisymmetry of $\mathbf{L}$ is then used to specify the elements $L_{\varepsilon \mathbf{D}}$ and $L_{\varepsilon \mathbf{B}}$. With this, the $\varepsilon$-component of the degeneracy requirement $\mathbf{L} \cdot(\delta S / \delta \mathbf{x})=\mathbf{0}$ can be satisfied by choosing $L_{\varepsilon \varepsilon}$ accordingly. Notice that this term is then also in consistence with the time

evolution for $\varepsilon$, and it is antisymmetric as well. The other two components of the degeneracy condition can be fulfilled by choosing $L_{\mathbf{D B}}=\nabla \times, L_{\mathbf{B D}}=-\nabla \times$, and $L_{\mathbf{D D}}=L_{\mathbf{B B}}=\mathbf{0}$. In 
summary, one obtains the Poisson operator

$$
\mathbf{L}=\left(\begin{array}{ccc}
\mathbf{E} \cdot \nabla \times \mathbf{H}-\mathbf{H} \cdot \nabla \times \mathbf{E} & -\mathbf{H} \cdot \nabla \times & \mathbf{E} \cdot \nabla \times \\
\nabla \times \mathbf{H} & \mathbf{0} & \nabla \times \\
-\nabla \times \mathbf{E} & -\nabla \times & \mathbf{0}
\end{array}\right)
$$

with all derivative operators acting on everything to the right, i.e., also on functions multiplied to the right side of the operator $\mathbf{L}$. At this point we mention that, in general, the symbol "." in (6) implies not only summation over discrete indices. If field variables are involved the operators $\mathbf{L}$ and $\mathbf{M}$ are written in terms of two space $\operatorname{arguments}\left(\mathbf{r}, \mathbf{r}^{\prime}\right)$, and an integration over $\mathbf{r}^{\prime}$ must be performed when multiplied with a function of $\mathbf{r}^{\prime}$ from the right. However, in the case of the field equations being local, one can express $\mathbf{L}$ and $\mathbf{M}$ in terms of a single variable $\mathbf{r}$ only [4], and no integration is implied when these operators are multiplied from the right. Such single variable notation is used for the Poisson operator (12)). However, in case of the friction matrix $\mathbf{M}$, discussed in the next section, it will be beneficial to keep the general form in terms of $\mathbf{r}$ and $\mathbf{r}^{\prime}$.

The reversible time evolution of the variables $\mathbf{x}$ as obtained from $\mathbf{L} \cdot \delta E / \mathbf{x}$ takes the form

$$
\begin{aligned}
\dot{\varepsilon}_{\mathrm{rev}} & =-\nabla \cdot(\mathbf{E} \times \mathbf{H}), \\
\dot{\mathbf{D}}_{\mathrm{rev}} & =\nabla \times \mathbf{H}, \\
\dot{\mathbf{B}}_{\mathrm{rev}} & =-\nabla \times \mathbf{E},
\end{aligned}
$$

in accord with (3) .

We note here that the lower-right $2 \times 2$ block matrix in L, Eq.(12), describing the electromagnetic part of the system, has been used previously by Marsden and Ratiu in [20]. Therein, it was given in terms of a Poisson bracket at which we can arrive by using $\{F, G\}=\int(\delta F / \delta \mathbf{x}) \cdot \mathbf{L} \cdot(\delta G / \delta \mathbf{x}) d^{3} r$. In our work, their Poisson bracket is embedded in a thermodynamic formulation with a thermal variable $\varepsilon$.

The degeneracy requirement and the antisymmetry of the Poisson operator are satisfied by construction. For the Jacobi identity, it is useful to observe that in our system the time evolution of the entropy density $s(\varepsilon, \mathbf{D}, \mathbf{B})$ has no reversible contributions. Since the transformation of variables does not affect the Jacobi identity, we choose to prove the Jacobi identity in the set of variables $\mathbf{x}^{\prime}=(s, \mathbf{D}, \mathbf{B})$, in which the Poisson operator $\mathbf{L}^{\prime}$ takes a much 
simpler form. Namely, only two elements, $L_{\mathbf{D B}}^{\prime}$ and $L_{\mathbf{B D}}^{\prime}$, are non-zero, and $\mathbf{L}^{\prime}$ coincides with the Poisson bracket in [20], for which the Jacobi identity is fulfilled. With this, the Poisson operator $\mathbf{L}$ is completely constructed.

\section{Friction matrix obtained from microscopic fluctuations}

Coarse graining with respect to time from one level of description (L1) to another one (L2) in principle leads to two kinds of irreversible effects on the coarser level. First, dissipative effects on level L1 reappear on level L2. Second, certain effects that are slower than the time resolution on level L1 and faster than the time resolution of L2 will be expressed as rapid fluctuations on level L2, and hence emerge as irreversible effects on that coarser level of description. It is this latter kind of irreversible effects after coarse-graining which we concentrate on. The coarse-graining procedure is captured in the following five steps and illustrated for the case of dissipative electromagnetism.

First, we express the friction matrix $\mathbf{M}$, which captures the dissipative contributions to the GENERIC evolution equation (6), in terms of a Green-Kubo-type expression. The matrix $\mathbf{M}$ can be calculated from the microscopic description by a generalization of the Green-Kubo relation for the viscosity (1). If $\dot{\mathbf{x}}^{\mathrm{f}}$ denotes the fluctuations on all components of $\mathbf{x}$, we write [4],

$$
\mathbf{M}\left(\mathbf{r}, \mathbf{r}^{\prime}\right)=\frac{1}{k_{\mathrm{B}}} \int_{0}^{\tau} d t\left\langle\dot{\mathbf{x}}^{\mathrm{f}}(\mathbf{r}, t) \dot{\mathbf{x}}^{\mathrm{f}, T}\left(\mathbf{r}^{\prime}, 0\right)\right\rangle
$$

which can be expressed alternatively, in component notation, as

$$
\left[1+\varepsilon\left(x_{i}\right) \varepsilon\left(x_{k}\right)\right] M_{i k}\left(\mathbf{r}, \mathbf{r}^{\prime}\right)=\frac{1}{k_{\mathrm{B}} \tau}\left\langle\triangle x_{i}^{\mathrm{f}}(\mathbf{r}) \triangle x_{k}^{\mathrm{f}}\left(\mathbf{r}^{\prime}\right)\right\rangle
$$

with $\varepsilon\left(x_{j}\right)= \pm 1$ depending on the parity of $x_{j}$ under time-reversal. Here, $\tau$ is an intermediate time scale separating the slow degrees of freedom (on the coarser level L2) from the fast ones (on the finer level L1). The quantity $\triangle x_{j}^{\mathrm{f}}(\mathbf{r})$ is given by $\triangle x_{j}^{\mathrm{f}}(\mathbf{r})=\int_{0}^{\tau} \dot{x}_{j}^{\mathrm{f}}(\mathbf{r}, t) d t$, and the brackets $\langle\ldots\rangle$ indicate the average over an ensemble of microscopic trajectories consistent with the slow macrostate $\mathbf{x}$ over the whole time interval, see pages 357-358 in [4] for more details. The superscript $T$ in (14) denotes the usual matrix transpose.

Second, for formulating the form of the fluctuations $\dot{\mathbf{x}}^{\mathrm{f}}$ the type of the variables $\mathbf{x}$ is important. Specifically, choosing densities of (conserved) extensive variables is particularly 
useful. Doing so is in close analogy to the common procedure in the theory of fluctuations in classical thermodynamics [1, 21]. Furthermore, the Green-Kubo relation (11), and similar relations for the thermal conductivity and diffusion coefficient, indicate that current-current correlations are quantities of fundamental importance. For example, the stress tensor is the current of momentum. Current densities play a major role in the formulation of conservation laws in the form of field equations for density variables of extensive quantities, e.g., internal energy density, momentum density. Hence, from this perspective it is tempting to use density variables in the set $\mathbf{x}$. How this can be exploited further is discussed in the third step below. In our example, the energy density $\varepsilon$ is hence a good variable, in contrast to the temperature $T$, or the (not conserved) entropy density $s$. Further dissipative processes we are considering here originate from the fluctuations in the particle positions and spins. Thus, fluctuations in $\mathbf{D}$ and $\mathbf{B}$ arise due to fluctuations in densities of extensive variables, namely in the polarization $\mathbf{P}$ and the magnetization $\mathbf{M}$.

Third, we make an ansatz for the structural form of the fluctuations. We assume that the evolution equations for the fluctuations are similar in structure to their macroscopic counterparts, in the spirit of Onsager's regression hypothesis. This hypothesis states that the fluctuations about the equilibrium state decay, on the average, according to the same laws that govern the decay of macroscopic deviations from equilibrium [2-4]. In view of the first component of $\dot{\mathbf{x}}^{\mathrm{f}}$ we note that (4) represents the change in electromagnetic energy only, while the corresponding equation for the thermal energy, $\varepsilon_{\mathrm{th}}$, of the body will be of the form $\dot{\varepsilon}_{\mathrm{th}}=\mathbf{J}_{e} \cdot \mathbf{E}-\nabla \cdot \mathbf{J}_{q}$ with heat flux $\mathbf{J}_{q}$, such that the total energy, i.e. the integral of $\varepsilon=\varepsilon_{\mathrm{em}}+\varepsilon_{\mathrm{th}}$, is conserved. Approximating the fluctuating contributions by white noise leads to expressions for $\Delta \mathrm{x}^{\mathrm{f}}$ of the form

$$
\Delta \mathbf{x}^{\mathrm{f}}=\left(\begin{array}{c}
-\nabla \cdot\left(\mathbf{W}^{(\mathrm{q})}+\mathbf{E} \times \mathbf{W}^{(\mathrm{H})}+\mathbf{W}^{(\mathrm{E})} \times \mathbf{H}\right) \\
\nabla \times \mathbf{W}^{(\mathrm{H})}-\mathbf{W}^{(\mathrm{j})} \\
-\nabla \times \mathbf{W}^{(\mathrm{E})}
\end{array}\right)
$$

where all $\mathbf{W}$ are (small) increments of Wiener processes, which are integrals of white noise over a time interval $\tau$. The superscripts to the Wiener processes indicate their physical origin.

Fourth, by specifying the second moments of the Wiener processes, and all correlations between them, the construction of the $\mathbf{M}$ matrix is completed. We assume that all $\mathbf{W}$ are 
decorrelated because of the difference in the underlying microscopic mechanisms, but that $\mathbf{W}^{\mathrm{q}}$ has a non-zero correlation only with the electric current fluctuations $\mathbf{W}^{\mathrm{j}}$, which will give rise to the thermoelectric effects. Hence we write the following relations

$$
\left\langle\mathbf{W}^{(\alpha)}(\mathbf{r}) \mathbf{W}^{(\beta)}\left(\mathbf{r}^{\prime}\right)\right\rangle=2 k_{\mathrm{B}} \tau \delta\left(\mathbf{r}-\mathbf{r}^{\prime}\right) \mathbf{C}^{(\alpha \beta)}(\mathbf{r}),
$$

with $\alpha, \beta \in\{\mathrm{q}, \mathrm{j}, \mathrm{E}, \mathrm{H}\}$, where the non-zero $\mathbf{C}^{(\alpha \beta)}$, i.e., $\mathbf{C}^{(\mathrm{qq})}, \mathbf{C}^{(\mathrm{jj})}, \mathbf{C}^{(\mathrm{EE})}, \mathbf{C}^{(\mathrm{HH})}$ and $\mathbf{C}^{(\mathrm{qj})} \equiv \mathbf{C}^{(\mathrm{jq}), T}$, represent the correlation functions, with implicit $\mathbf{r}$-dependence, e.g., through temperature. Note that we have assumed only local correlations between the Wiener processes, i.e., between the fluctuations. Hence, we are dealing with a local field formulation. However, non-local effects can be included if so desired.

In the fifth and final step, the firction matrix $\mathbf{M}$ is calculated. Using the fluctuations (16) in conjunction with the correlations (17) we calculate the Green-Kubo relations for the elements of the matrix $\mathbf{M}\left(\mathbf{r}, \mathbf{r}^{\prime}\right)$ by way of (15)). The final result for the friction matrix is

$$
\mathbf{M}\left(\mathbf{r}, \mathbf{r}^{\prime}\right)=\left(\begin{array}{ccc}
M_{\varepsilon \varepsilon} & M_{\varepsilon \mathbf{D}} & M_{\varepsilon \mathbf{B}} \\
M_{\mathbf{D} \varepsilon} & M_{\mathbf{D D}} & 0 \\
M_{\mathbf{B} \varepsilon} & 0 & M_{\mathbf{B B}}
\end{array}\right),
$$

with

$$
\begin{aligned}
M_{\varepsilon \varepsilon}= & \nabla \cdot \mathbf{C}^{(\mathrm{qq})}(\mathbf{r}) \cdot\left(\nabla^{\prime} \delta\left(\mathbf{r}-\mathbf{r}^{\prime}\right)\right)+\nabla \cdot \mathbf{E}(\mathbf{r}) \times \mathbf{C}^{(\mathrm{HH})}(\mathbf{r}) \cdot\left(\nabla^{\prime} \times \mathbf{E}\left(\mathbf{r}^{\prime}\right) \delta\left(\mathbf{r}-\mathbf{r}^{\prime}\right)\right) \\
& +\nabla \cdot \mathbf{H}(\mathbf{r}) \times \mathbf{C}^{(\mathrm{EE})}(\mathbf{r}) \cdot\left(\nabla^{\prime} \times \mathbf{H}\left(\mathbf{r}^{\prime}\right) \delta\left(\mathbf{r}-\mathbf{r}^{\prime}\right)\right) \\
M_{\mathbf{D D}}= & -\nabla \times \mathbf{C}^{(\mathrm{HH})}(\mathbf{r}) \cdot\left(\nabla^{\prime} \delta\left(\mathbf{r}-\mathbf{r}^{\prime}\right)\right) \times+\delta\left(\mathbf{r}-\mathbf{r}^{\prime}\right) \mathbf{C}^{(\mathrm{jj})}(\mathbf{r}) \\
M_{\mathbf{B B}}= & -\nabla \times \mathbf{C}^{(\mathrm{EE})}(\mathbf{r}) \cdot\left(\nabla^{\prime} \delta\left(\mathbf{r}-\mathbf{r}^{\prime}\right)\right) \times \\
M_{\varepsilon \mathbf{D}}= & \nabla \cdot \mathbf{C}^{(\mathrm{qj})}(\mathbf{r}) \delta\left(\mathbf{r}-\mathbf{r}^{\prime}\right)+\nabla \cdot \mathbf{E}(\mathbf{r}) \times \mathbf{C}^{(\mathrm{HH})}(\mathbf{r}) \cdot\left(\nabla^{\prime} \delta\left(\mathbf{r}-\mathbf{r}^{\prime}\right)\right) \times \\
M_{\mathbf{D} \varepsilon}= & \mathbf{C}^{(\mathrm{qj}), T}(\mathbf{r}) \cdot\left(\nabla^{\prime} \delta\left(\mathbf{r}-\mathbf{r}^{\prime}\right)\right)-\nabla \times \mathbf{C}^{(\mathrm{HH})}(\mathbf{r}) \cdot\left(\nabla^{\prime} \times \mathbf{E}\left(\mathbf{r}^{\prime}\right) \delta\left(\mathbf{r}-\mathbf{r}^{\prime}\right)\right) \\
M_{\varepsilon \mathbf{B}}= & \nabla \cdot \mathbf{H}(\mathbf{r}) \times \mathbf{C}^{(\mathrm{EE})}(\mathbf{r}) \cdot\left(\nabla^{\prime} \delta\left(\mathbf{r}-\mathbf{r}^{\prime}\right)\right) \times \\
M_{\mathbf{B} \varepsilon}= & -\nabla \times \mathbf{C}^{(\mathrm{EE})}(\mathbf{r}) \cdot\left(\nabla^{\prime} \times \mathbf{H}\left(\mathbf{r}^{\prime}\right) \delta\left(\mathbf{r}-\mathbf{r}^{\prime}\right)\right)
\end{aligned}
$$

where it is understood that contractions . and cross products $\times$ are executed in the order of occurrence from the right to the left.

Given the expression (18) for $\mathbf{M}$, it is straightforward to show that the degeneracy condition $\mathbf{M} \cdot(\delta E / \delta \mathbf{x})=\mathbf{0}$ is indeed satisfied. The origin of this result goes back to the ansatz 
for the fluctuations $\Delta \mathrm{x}^{\mathrm{f}}$ in (16). Their specific form ensures that the fluctuations occur on a submanifold of constant energy, as can be shown readily by

$$
\int \Delta \mathbf{x}^{\mathrm{f}, T} \cdot \frac{\delta E}{\delta \mathbf{x}} d^{3} r=0
$$

as required by the GENERIC. Equation (19) is fulfilled already by writing the first component of $\dot{\mathbf{x}}^{\mathrm{f}}$, namely the fluctuations in the total energy density, as in (16), which requires it to be the divergence of a vector field. It is then straightforward to show that (19) is satisfied if boundary terms can be neglected.

If dissipative matrix $\mathbf{M}\left(\mathbf{r}, \mathbf{r}^{\prime}\right)$ is applied to the entropy gradient $\delta S / \delta \mathbf{x}\left(\mathbf{r}^{\prime}\right)$, including an integration over $\mathbf{r}^{\prime}$, one gets the irreversible contributions to the time evolution equations. Using the definitions

$$
\begin{aligned}
\mathbf{E}^{*} & =\frac{1}{T} \mathbf{C}^{(\mathrm{EE})} \cdot(\nabla \times \mathbf{H}), \\
\mathbf{H}^{*} & =-\frac{1}{T} \mathbf{C}^{(\mathrm{HH})} \cdot(\nabla \times \mathbf{E}), \\
\mathbf{J}_{e}^{*} & =\mathbf{C}^{(\mathrm{jj})} \cdot \frac{\mathbf{E}}{T}+\mathbf{C}^{(\mathrm{qj}), T} \cdot \nabla \frac{1}{T},
\end{aligned}
$$

one obtains for the irreversible part of the time evolutions of $\mathbf{D}$ and $\mathbf{B}$

$$
\begin{aligned}
& \dot{\mathbf{D}}_{\text {irr }}=\nabla \times \mathbf{H}^{*}-\mathbf{J}_{e}^{*}, \\
& \dot{\mathbf{B}}_{\text {irr }}=-\nabla \times \mathbf{E}^{*},
\end{aligned}
$$

where all functions are evaluated at $\mathbf{r}$. We recognize in $\dot{\mathbf{D}}_{\mathrm{irr}}$ the Ohmic conduction $\mathbf{C}^{(\mathrm{jj})}$, while $\mathbf{C}^{(\mathrm{q} j)}$ represents the thermoelectric coupling [1].

\section{E. Final set of evolution equations}

The final electromagnetic time evolution equations can be conveniently written in the following way. Combining the reversible (13) and irreversible (21) contributions to the time evolution equations of the fields $\mathbf{D}$ and $\mathbf{B}$, the resulting temporally coarse-grained Maxwell equations differ from (3) in that the fields $\mathbf{E}, \mathbf{H}$ and $\mathbf{J}_{e}$ in (3) have to be replaced by $\mathbf{E}+\mathbf{E}^{*}$, $\mathbf{H}+\mathbf{H}^{*}$ and $\mathbf{J}_{e}+\mathbf{J}_{e}^{*}$, respectively. The additional fields $\mathbf{E}^{*}, \mathbf{H}^{*}$ and $\mathbf{J}_{e}^{*}$ give rise to the irreversible contributions to $\dot{\mathbf{D}}$ and $\dot{\mathbf{B}}$. They are proportional to the quantities $\nabla \times \mathbf{H}$, $\nabla \times \mathbf{E}, \mathbf{E}$ and $\nabla T$, as expected from conditions (5) and the discussion in Sec. II, and 
represent the nonequilibrium forces that tend to restore equilibrium. We mention that the full macroscopic Maxwell equations obtained here can be used to derive expressions for the complex permittivity $\boldsymbol{\epsilon}(\omega)$ and permeability $\boldsymbol{\mu}(\omega)$ when examined in the linear response regime [6, 8]].

The frequently considered balance equation for the energy density can be obtained from (13a) and the result of the application of $\mathbf{M}\left(\mathbf{r}, \mathbf{r}^{\prime}\right)$ to the entropy gradient $\delta S / \delta \mathbf{x}\left(\mathbf{r}^{\prime}\right)$, namely,

$$
\dot{\varepsilon}=-\nabla \cdot(\mathbf{E} \times \mathbf{H})-\nabla \cdot\left(\mathbf{E} \times \mathbf{H}^{*}+\mathbf{E}^{*} \times \mathbf{H}+\mathbf{C}^{(\mathrm{qq})} \cdot \nabla \frac{1}{T}+\mathbf{C}^{(\mathrm{qj})} \cdot \frac{\mathbf{E}}{T}\right) .
$$

The first term on the right side, which is the divergence of the well known Poynting vector, represents the reversible part of the time evolution equation, while the second term is the irreversible part, describing the heat conduction (through $\mathbf{C}^{(\mathrm{qq})}$ ) and the Peltier effect (through $\mathbf{C}^{(\mathrm{qj})}$ ), as well as a modification of the electromagnetic energy flux, i.e., of the Poynting vector, due to the additional fields $\mathbf{E}^{*}$ and $\mathbf{H}^{*}$. This modification of the energy flux was found also by Liu [7, 9].

For the time evolution of the entropy density $s$ one can write, using the chain rule and rearranging the expression,

$$
\begin{aligned}
\dot{s} & =-\nabla \cdot\left(\frac{1}{T} \mathbf{C}^{(\mathrm{qq})} \cdot \nabla \frac{1}{T}+\frac{1}{T} \mathbf{C}^{(\mathrm{qj})} \cdot \frac{\mathbf{E}}{T}\right) \\
& +\left(\begin{array}{c}
\nabla \frac{1}{T} \\
\frac{\mathbf{E}}{T} \\
\frac{1}{T}(\nabla \times \mathbf{E}) \\
\frac{1}{T}(\nabla \times \mathbf{H})
\end{array}\right) \cdot\left(\begin{array}{cccc}
\mathbf{C}^{(\mathrm{qq})} & \mathbf{C}^{(\mathrm{qj})} & \mathbf{0} & \mathbf{0} \\
\mathbf{C}^{(\mathrm{qj}), T} & \mathbf{C}^{(\mathrm{jj})} & \mathbf{0} & \mathbf{0} \\
\mathbf{0} & \mathbf{0} & \mathbf{C}^{(\mathrm{HH})} & \mathbf{0} \\
\mathbf{0} & \mathbf{0} & \mathbf{0} & \mathbf{C}^{(\mathrm{EE})}
\end{array}\right) \cdot\left(\begin{array}{c}
\nabla \frac{1}{T} \\
\frac{\mathbf{E}}{T} \\
\frac{1}{T}(\nabla \times \mathbf{E}) \\
\frac{1}{T}(\nabla \times \mathbf{H})
\end{array}\right) .
\end{aligned}
$$

The central matrix of the last term is positive semidefinite as can be seen after inserting the microscopic expressions for the correlation functions (17), and therefore the entropy production rate is indeed non-negative. The obtained entropy production rate corresponds to the one proposed by Liu in [5, 6].

\section{DISCUSSION AND CONCLUSIONS}

Coarse-grained dissipative Maxwell equations of electromagnetism have been obtained and examined from the perspective of nonequilibrium thermodynamics by using the GENERIC formalism. We have illustrated a scheme to relate the dissipative effects on 
the macroscopic scale to the fast microscopic fluctuations in the polarization and magnetization in the spirit of a generalized fluctuation-dissipation theorem. In particular, the study is applicable also to materials having nonlinear constitutive relations between $(\mathbf{E}, \mathbf{B})$ and $(\mathbf{D}, \mathbf{H})$. It emerged clearly that the difference between spatial and temporal coarse-graining is of fundamental importance. On the one hand, the usual procedure of averaging the microscopic Maxwell equations only spatially (or by single-time ensembles averages) leads to terms equivalent to the reversible contributions in the GENERIC formulation, $\mathbf{L} \cdot(\delta E / \delta \mathbf{x})$. On the other hand, coarse-graining the spatially averaged Maxwell equations further with respect to time leads to new irreversible effects captured in terms of $\mathbf{M} \cdot(\delta S / \delta \mathbf{x})$, as shown here. The friction matrix $\mathbf{M}$ has been related to two-time correlations of fast processes on the finer level of description. Using the Green-Kubo expression (14), the correlations of microscopic fluctuations give rise to dissipative processes such as Ohmic currents, the thermoelectric effect, and to other irreversible contributions to the electric and magnetic fields.

In the above investigations, the following points have to be highlighted. First, we presented an illustration of the coarse-graining procedure in order to obtain the structure of fluctuations and, in turn, the structure of the dissipative effects in electromagnetism. Second, the identification of current densities occurring in $\dot{\mathbf{x}}^{\mathrm{f}}$ was of fundamental importance, since correlations between them lead to the new dissipative processes on the macroscopic scale. To aid the relation between the Green-Kubo-type expression for $\mathbf{M}$ (14) and the correlations between current densities, it has proven useful to choose density variables of extensive quantities in the set $\mathbf{x}$. Using Onsager's regression hypothesis, the evolution equations of the fluctuations takes the form of conservation laws, including fluctuating current densities. We point out that the correlations (17) are similar in spirit to the Green-Kubo relation (11), in the sense that they define transport coefficients. In contrast, the expression for $\mathbf{M}$ (14) in conjunction with the entropy gradient leads to the full form of the dissipative processes. The third point concerns the details behind the correlations (17). In order to specify the dissipative processes beyond their structure, one needs to discuss in more detail the physics behind the correlation matrices $\mathbf{C}^{\alpha \beta}$, with $\alpha, \beta \in\{\mathrm{q}, \mathrm{j}, \mathrm{E}, \mathrm{H}\}$. That is, the transport coefficients appearing in the final expressions through $\mathbf{C}^{\alpha \beta}$ can be obtained, e.g., via molecular dynamics simulations, or be derived based on explicit expressions for the microscopic fluxes and the correlations between them [22]. 
The scheme of temporal coarse-graining for obtaining the macroscopic Maxwell equations differs from Liu's [5, 6], who started by making an ansatz for the entropy production rate. However, both procedures lead to identical modifications of the Maxwell equations, and also the energy equation (22) is in agreement with the corresponding relations in [5, 6]. The obtained Maxwell relations and extensions of them are successfully applied to the problems of nematic liquid crystals [5, 6], colloidal magnetic and electric fluid, and ferrofluids [79]. In nematic liquid crystals, the dissipative couplings between the new thermodynamic forces $\nabla \times \mathbf{E}$ and $\nabla \times \mathbf{H}$ with the additional thermodynamic forces specific for this system are permitted and are equipped with transport coefficients, thus being valid also in case of the oscillatory instabilities, when usually employed static Maxwell equations no longer hold [5, 6]. Liu's equations for ferrofluids also give rise to the dissipative forces that account for the special spin-up behavior of ferrofluids under a rotating external field [7-9].

A cross relationship, similar to the one suggested by Liu [5], occurred in the dissipative terms (21) obtained by us: $\mathbf{C}^{(\mathrm{HH})}$ in $\dot{\mathbf{D}}_{\text {irr }}$ accounts for the correlations of the fluctuations in magnetization, i.e. the magnetization relaxation time, and $\mathbf{C}^{(\mathrm{EE})}$ in $\dot{\mathbf{B}}_{\text {irr }}$ accounts for the correlations of the fluctuations in polarization, i.e. the polarization relaxation time. This is the consequence of the nature of Maxwell equations and of the mutual dependence of the electric and magnetic fields. It is naturally obtained by the form (16) for the increments in fluxes $\Delta \mathbf{x}^{\mathrm{f}}$.

Aspects, different from the ones discussed here, of dissipative effects in electromagnetism and of linking electromagnetism with hydrodynamics can be found in [1, 19, 23]. Decomposition of the electromagnetic fields, in particular polarization and magnetization, into the slow and fast parts has been considered by Felderhof and Kroh [19]. In this way they extended the irreversible thermodynamics approach used by de Groot and Mazur [1] for the hydrodynamics of magnetic and dielectric fluids in interaction with the electromagnetic field. In a subsequent paper [23], Felderhof studied semirelativistic hydrodynamic evolution equations for a medium with polarization and magnetization.

[1] S.R. de Groot and P. Mazur, Non-equilibrium Thermodynamics (North-Holland, Amsterdam, 1962) 
[2] R. Kubo, M. Toda and N. Hashitsume, Nonequilibrium Statistical Mechanics, vol. II of Statistical Physics (Springer, Berlin, 1991) 2nd edition

[3] D.J. Evans and G.P. Morriss, Statistical Mechanics of Nonequilibrium Liquids (Academic Press, 1990)

[4] H.C. Öttinger, Beyond Equilibrium Thermodynamics (Wiley, Hoboken, N.J., 2005)

[5] M. Liu, Phys. Rev. Lett. 70, 3580 (1993)

[6] M. Liu, Phys. Rev. E 50, 2925 (1994)

[7] M. Liu, Phys. Rev. Lett. 74, 4535 (1995)

[8] M. Liu, Phys. Rev. Lett. 80, 2937 (1998)

[9] M. Liu, Phys. Rev. E 59, 3669 (1999)

[10] M. Liu, Physik Journal 1(12), 49 (2002)

[11] Y. Jiang and M. Liu, Phys. Rev. Lett. 77, 1043 (1996)

[12] M. Grmela and H.C. Öttinger, Phys. Rev. E 56, 6620 (1997)

[13] H.C. Öttinger and M. Grmela, Phys. Rev. E 56, 6633 (1997)

[14] J.D. Jackson, Classical Electrodynamics (Wiley, New York, 1975)

[15] F.N.H. Robinson, Macroscopic Electromagnetism (Pergamon, Oxford, 1973)

[16] S.R. de Groot and L.G. Suttorp, Foundations of Electrodynamics (North-Holland, Amsterdam, 1972)

[17] H.C. Öttinger, Hydrodynamics from Boltzmann's Kinetic Equation after Proper CoarseGraining, Preprint, submitted to Phys. Rev. E (2005).

[18] L.D. Landau and E.M. Lifshitz, Electrodynamics of Continuous Media (Pergamon, Oxford, 1984)

[19] B.U. Felderhof and H.J. Kroh, J. Chem. Phys. 110, 7403 (1999)

[20] J.E. Marsden and T.S. Ratiu, Mechanics and Symmetry (Springer, New York, 1999)

[21] H.B. Callen, Thermodynamics and an Introduction to Thermostatistics (2nd. ed.) (Wiley, New York, 1985)

[22] P. Español and C. Thieulot, J. Chem. Phys. 118, 9109 (2003)

[23] B.U. Felderhof, J. Chem. Phys. 120, 3598 (2004) 